\title{
A duration approach for the measurement of biometric risks in life insurance
}

\author{
Marius Radermacher (D) Peter Recht
}

\begin{abstract}
Duration concepts are standard methods for measuring interest rate risks of portfolios, liabilities or other cash flows. Macaulay duration, effective duration and key-rate duration are widely used in practice according to different types of yield curves. In this paper, we will present a formulation for a forward rate duration measure by using multi-dimensional Taylor series approximation. It allows to measure the interest rate risk based on arbitrary forward rates. This approach can easily be adopted for biometric risk management, allowing the definition of a biometric duration. The biometric duration will be applied to actuarial present values of premiums and benefits as well as the actuarial reserve to quantify the corresponding biometric risk. It proves to be an easy-to-use tool in the actuarial practise.
\end{abstract}

Keywords Duration measure $\cdot$ Biometric risk $\cdot$ Forward rates $\cdot$ Biometric duration

M. Radermacher $(\bowtie) \cdot$ P. Recht

Technical University Dortmund, Vogelpothsweg 87, 44227 Dortmund, Germany

E-Mail: marius.radermacher@tu-dortmund.de

P. Recht

E-Mail: peter.recht@tu-dortmund.de 


\section{Ein Durationsansatz für die Messung biometrischer Risiken in der Lebensversicherung}

Zusammenfassung Durationskonzepte zählen zu den Standardmethoden bei der Messung von Zinsänderungsrisiken von Zahlungsreihen. In der Praxis werden häufig die Macaulay-Duration, die effektive Duration und die Key-Rate-Duration, entsprechend ihrer zugrundeliegenden Zinskurven, angewendet. In diesem Beitrag stellen wir eine Formulierung für eine Forward-Rate-Duration unter Verwendung einer mehrdimensionalen Taylor- Reihenentwicklung vor. Sie ermöglicht die Messung des Zinsänderungsrisikos auf der Grundlage beliebiger Terminzinsen. Dieser Ansatz lässt sich leicht auf die Messung biometrischer Risiken anwenden und ermöglicht die Definition einer biometrischen Duration. Die biometrische Duration wird auf die versicherungsmathematischen Barwerte der Prämien und Leistungen sowie auf die Deckungsrückstellung zur Quantifizierung des entsprechenden biometrischen Risikos angewendet. Es erweist sich als ein Instrument, das auf einfache Art und Weise in der aktuariellen Praxis angewendet werden kann.

Schlüsselwörter Durationsmaß · Biometrisches Risiko · Forward Rates · Biometrische Duration

\section{Introduction}

Interest rates have a significant impact on the present value of a cash flow. Especially cash flows with long term maturities like investments, actuarial premiums and benefits etc. are sensitive to any shift of the corresponding yield curve. Therefore, perturbations in the yield curve affect the present value of these cash flows and imply an interest rate risk. Identifying, measuring and controlling interest rate risk exposure is a key task of risk management (see e.g. Bühler and Hies 1995; Fabozzi et al. 2003; Ho 1992). Especially in financial markets with high interest rate volatility, it is mandatory to examine the effects of interest rate changes.

In practical risk management, the probably most commonly used measures are the duration measures. The duration measure was introduced by Macaulay (1938) and originally defined as the average remaining time of a cash flow. It is further modified and extended for measuring the sensitivity with respect to changes in the yield curve by Hicks (1939). By this modified duration the interest rate risk can be estimated, if a flat yield curve is applied. Fisher and Weil (1971) developed an optimal immunization strategy for bond investments based on such durations. Taking into account the term structure of interest rates, their effective duration (also named Fisher-Weil duration) can be used to measure the sensitivity of a parallel shift of a spot rate yield curve. Furthermore, Ho (1992) introduced an instrument to measure the sensitivity of a security to each key rate change. By the key rate durations the effects of non-parallel shifts of the corresponding curve can be quantified. Considering an arbitrary type of interest rate change, Fong and Vasicek (1984) presented a strategy to minimize the immunization risk. 
In addition to financial parameters, life insurers must also take biometric data into account. Mortality rates are used to consider the uncertainty of the occurrence of survival and death benefits. For practical life insurance, a deterministic model of mortality rates is used. Although mortality rates are considered deterministic, their values are stochastic and change over time. The risk that mortality rates may deviate from the expected ones is known as the biometric risk. It covers the longevity risk and the mortality risk. Both terms are used in literature for the risk coming from an increase or decrease of the life expectancy of pensioners, respectively.

It is widely accepted that this type of risk also plays an important role in life insurance as it influences the calculation of premiums, benefits and reserves. Hence, there is need for an adequate consideration of biometric risks (see e.g. Cairns et al. 2006; Plat 2011).

One way of dealing with biometric risk is to model mortalities as stochastic processes. They mostly focus on a precise prediction of trend in mortality. The techniques that are involved come from frameworks used for the prediction of interest rates. For a review of such stochastic models see e.g. Cairns et al. (2006).

On the other hand, there are deterministic approaches dealing with biometric risk. Rather than investigating proper trends of mortality, the effects of simulated changes in mortality are examined.

Inspired by interest rate risk theory, Coughlan et al. (2007) and Coughlan (2012) proposed the term $q$-duration, to denote a technique for measuring the mortality sensitivity. Wang et al. (2010) adopted the concept of effective duration to mortality. Plat (2011) implemented Wangs $q$-duration and $q$-convexity and gave formulas for considering a parallel shift of the life table.

However, Plat has also recognized difficulties to determine such a shift since changes in mortalities are different for different ages and future years. Li and Luo (2012), therefore, assumes that mortality curves are subject to non-parallel shifts. Accordingly to Ho's key rate duration, Li and Luo extended the $q$-duration to the key $q$-duration. From this, they propose an optimal strategy for hedging the longevity risk.

Overall, for the financial sector, the duration concept already provides a number of instruments used in risk management. The various duration measures serve to analytically identify and interpret interest rate risks in a simple manner. They represent tools that are indispensable in the daily practice of financial risk management.

It is desirable that a similar concept for biometric risks is available. The requirements that Solvency II, for example, imposes on the constant monitoring of risks make it necessary to have similar "easy-to-use" instruments for biometrics.

With $q$-duration and key $q$-duration, there are attempts to transfer elements from the classical duration concept to the management of biometric risks. But here the underlying ideas are either sketchy or inconvenient. Therefore, these instruments are less suitable for daily use in an actuarial environment.

In the following, an instrument shall be presented that an actuary can use during his daily work in the management of biometric risks. The easy-to-use instrument we propose, is based on the fundamental ideas of existing duration concepts. Looking at the different duration measures from a somewhat more general perspective, they turn out to be special cases of a so-called forward rate duration. It is decisive that this 
forward rate duration can easily be adopted so that biometric risk can be measured accurately and efficiently. This makes it suitable for daily practical use.

The paper is organized as follows. In Sects. 2 and 3 we recall some basics. The most common duration measures for the interest rate risk, modified duration and key rate duration, will be reviewed in order to identify their analytical similarities. This will help to define the forward rate duration in Sect. 4 for financial use. Sect. 5 adapts this idea for actuarial purposes. We will give examples of the practical use of the biometric duration and apply it to different types of life insurance contracts. The practical significance and applicability of the biometric duration is illustrated. We will conclude with a summary in Sect. 6.

\section{Preliminaries}

We consider a time horizon of $n$ periods $[t-1, t], t=1, \ldots, n$ and payments $Z_{t}$ due at time $t=0,1, \ldots, n$. The corresponding cash flow is denoted by $Z=\left(Z_{0}, Z_{1}, \ldots, Z_{n}\right)$. We will evaluate $Z$ by its present value with either a flat yield curve, spot rates or forward rates.

A flat yield curve $\bar{I}=(i, i, \ldots, i)$ is induced by a constant interest rate $i>-1$. The present value $P V(\bar{I})^{1}$, depending on this constant interest $i$, is given by

$$
P V(Z, \bar{I})=\sum_{j=0}^{n} Z_{j} \cdot(1+i)^{-j} .
$$

In a more general situation spot rates $i_{[0, t]}>-1, t=1, \ldots, n$, are considered for each respective maturity by a yield curve $I^{s}=\left(i_{[0,1]}, i_{[0,2]}, \ldots, i_{[0, n]}\right)$. In this case, the present value of $Z$ equals

$$
P V\left(Z, I^{S}\right)=\sum_{j=0}^{n} Z_{j} \cdot\left(1+i_{[0, j]}\right)^{-j} .
$$

As a third variant forward rates $i_{t}:=i_{[t-1, t]}>-1$ are assumed for each period $[t-1, t], t=1, \ldots, n$. The corresponding forward rate yield curve is denoted by $I^{f}=\left(i_{1}, i_{2}, \ldots, i_{n}\right)$. The discount factor for a payment at time $t$, evaluated at time $k$ is denoted by

$$
v_{k}^{(t)}:=\prod_{j=k+1}^{t}\left(1+i_{j}\right)^{-1}
$$

\footnotetext{
${ }^{1}$ A present value depends on $Z, n$ and the interest rates used. The notation $P V(\cdot)$ is used to identify the present values according to the respective interest rate curves.
} 

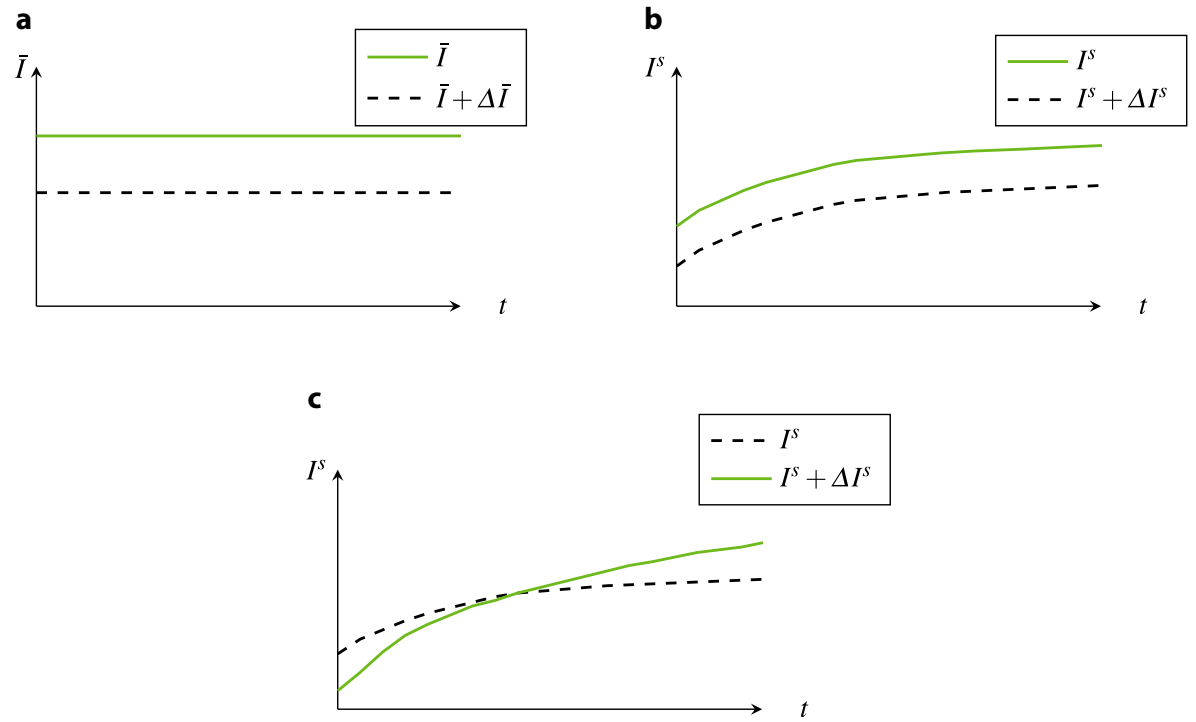

Fig. 1 Examples of different shifts of yield curves. a Parallel shift of a flat yield curve. b Parallel shift of a non-flat yield curve. c Non-parallel shift of a non-flat yield curve

For $k=0$, the notation $v_{0}^{(t)}=v^{(t)}$ is used. Hence,

$$
P V\left(Z, I^{f}\right)=\sum_{j=0}^{n} Z_{j} \cdot v^{(j)} .
$$

Remark 1 The yield curves $I^{s}, I^{f}$ and $\bar{I}$ obviously coincide, if $i_{[0, t]}=i_{t}=\mathrm{i}$ for all $t=1, \ldots, n$.

Furthermore, if spot rates $i_{[0, t]}$ are given for $t=1, \ldots, n$ related forward rates $i_{\mathrm{t}}$ can formally be extracted via

$$
i_{t}= \begin{cases}i_{[0,1]}, & t=1, \\ \left(1+i_{[0, t]}\right)^{t} \cdot\left(1+i_{[0, t-1]}\right)^{-(t-1)}-1, & t=2, \ldots, n .\end{cases}
$$

Of course, $P V\left(Z, I^{f}\right)=P V\left(Z, I^{s}\right)$ holds in this case.

\section{Duration concepts in interest rate risk management}

The interest rate risk of $Z$ arises by a change of the present value as interest rates change. The sensitivity of a present value is represented by its relative change caused by a change in interest rates. Hence, sensitivity is a relative measurement of the interest rate risk.

Changes of flat yield curves $\bar{I}$ correspond to parallel shifts, i.e. $\bar{I}+\Delta \bar{I}=$ $(i, i, \ldots, i)+(\Delta i, \Delta i, \ldots, \Delta i)$ (see Fig. 1a), with $i+\Delta i>-1$. 
Fig. 2 Approximation error caused by the first order approximation and the second order approximation

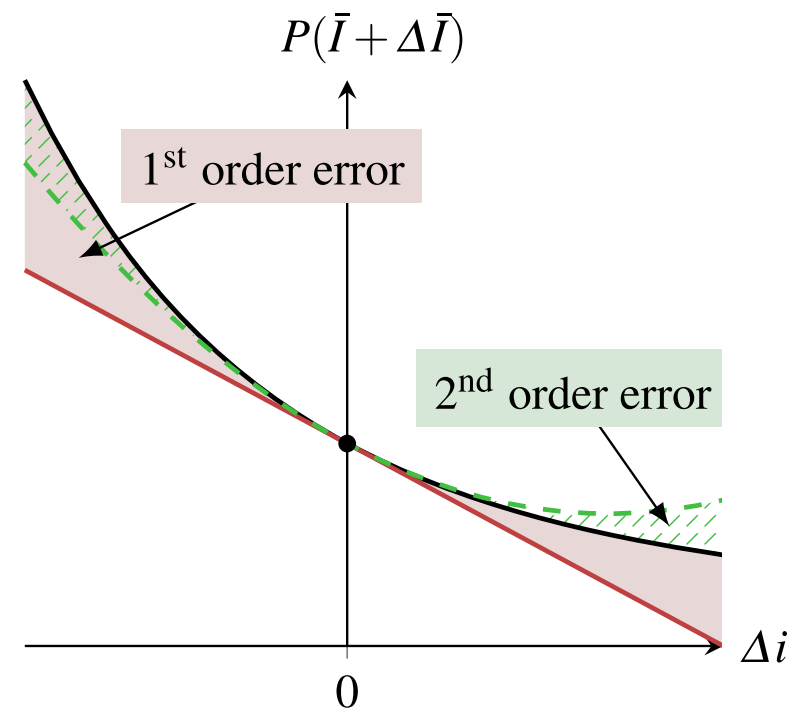

In this case, the interest rate risk can be approximated by a Taylor series expansion (see e.g. Tuckman and Serrat 2011). The first-order approximation

$$
P V(Z, \bar{I}+\Delta \bar{I})-P V(Z, \bar{I}) \approx \frac{\mathrm{d} P V}{\mathrm{~d} i} \Delta i
$$

leads to an approximation of the sensitivity

$$
\frac{\Delta P V(Z, \bar{I})}{P V(Z, \bar{I})}=\frac{P V(Z, \bar{I}+\Delta \bar{I})-P V(Z, \bar{I})}{P V(Z, \bar{I})} \approx-\left(\frac{-1}{P V(Z, \bar{I})} \frac{\mathrm{d} P V}{\mathrm{~d} i}\right) \Delta i .
$$

The quanitity

$$
\begin{aligned}
& D(Z, \bar{I}):=\frac{-1}{P V(Z, \bar{I})} \frac{\mathrm{d} P V}{\mathrm{~d} i} \\
& =\frac{1}{P V(Z, \bar{I})}\left((1+i)^{-1} \cdot \sum_{j=1}^{n} j \cdot Z_{j} \cdot(1+i)^{-j}\right) .
\end{aligned}
$$

corresponds to the modified duration ${ }^{2}$ (see e.g. Bühler and Hies 1995). As a function of $\Delta i$, the approximation of $P V(Z, \bar{I}+\Delta \bar{I})$ changes linearly in $\Delta i$. Hence, the approximation error is small only for small parallel shifts $\Delta i$ (see Fig. 2).

The second-order Taylor approximation generally leads to an improvement of the approximation, i.e.

$$
P V(Z, \bar{I}+\Delta \bar{I})-P V(Z, \bar{I}) \approx \frac{\mathrm{d} P V}{\mathrm{~d} i} \Delta i+\frac{1}{2} \frac{\mathrm{d}^{2} P V}{\mathrm{~d} i^{2}} \Delta i^{2}
$$

\footnotetext{
${ }^{2}$ For the modified duration $D(Z, \bar{I})$ the notation $D$ or $D(i)$ is often used in standard literature.
} 
and

$$
\frac{\Delta P V(Z, \bar{I})}{P V(Z, \bar{I})} \approx \frac{1}{P V(Z, \bar{I})} \frac{\mathrm{d} P V}{\mathrm{~d} i} \Delta i+\frac{1}{2 P V(Z, \bar{I})} \frac{\mathrm{d}^{2} P V}{\mathrm{~d} i^{2}} \Delta i^{2} .
$$

The approximation error in this case is usually smaller, even for larger values of $\Delta i$, see Fig. 2. The expression

$$
\begin{aligned}
C(Z, \bar{I}) & :=\frac{1}{P V(Z, \bar{I})} \frac{\mathrm{d}^{2} P V}{\mathrm{~d} i^{2}} \\
& =\frac{(1+i)^{-2}}{P V(Z, \bar{I})} \cdot\left(\sum_{j=1}^{n} j \cdot(j+1) \cdot Z_{j} \cdot(1+i)^{-j}\right)
\end{aligned}
$$

is usually called convexity (see e.g. Trautmann 2007). Using $D(Z, \bar{I})$ and $C(Z, \bar{I})$ the sensitivity in (7) can be formulated as

$$
\frac{\Delta P V(Z, \bar{I})}{P V(Z, \bar{I})} \approx-D(Z, \bar{I}) \cdot \Delta i+\frac{1}{2} \cdot C(Z, \bar{I}) \cdot \Delta i^{2} .
$$

Not only taking parallel shifts into account, but also the consideration of flat yield curves themselves is already very restrictive within real world environments. Hence, $D(Z, \bar{I})$ and $C(Z, \bar{I})$ are not satisfactory to model interest rate risks appropriately. A non-flat yield curve is, therefore, often considered for practical purposes. Fisher and Weil (1971) refined $D(Z, \bar{I})$ taking into account the use of a spot rate yield curve $I^{s}$, but still assuming parallel shifts $\Delta \bar{I}$ (see Fig. 1b)

$$
I^{s}+\Delta \bar{I}=\left(i_{[0,1]}, i_{[0,2]}, \ldots, i_{[0, n]}\right)+(\Delta i, \Delta i, \ldots, \Delta i) .
$$

But usually the disturbances in interest rates over time are not constant. Shortterm interest rates are typically more volatile than long-term interest rates and the former usually reach higher levels (Schich 1997). It is, obviously, more realistic to take into account non-parallel shifts of the yield curve (An example is shown in Fig. 1b). For non-parallel shifts of a spot rate yield curve $I^{S}$ the so-called key rate durations can be applied (Ho 1992). The $t$-key rate duration quantifies the sensitivity with respect to a change $\Delta i_{[0, t]}$ of a (single) spot rate $i_{[0, t]}$, i.e.

$$
\begin{aligned}
K R D_{t} & =\frac{-1}{P V\left(Z, I^{s}\right)} \cdot \frac{\mathrm{d} P V\left(Z, I^{s}\right)}{\mathrm{d} i_{[0, t]}} \\
& =\frac{t \cdot Z_{t} \cdot\left(1+i_{[0, t]}\right)^{-(t+1)}}{P V\left(Z, I^{s}\right)} .
\end{aligned}
$$


Note, that

$$
D\left(Z, I^{s}\right)=\sum_{j=1}^{n} K R D_{j}
$$

holds. Within a Taylor approximation of the present value $P V\left(Z, I^{s}\right)$ the key rate durations appear

$$
\Delta P V\left(Z, I^{s}\right) \approx-P V\left(Z, I^{s}\right) \cdot \sum_{j=1}^{n} K R D_{j} \cdot \Delta i_{[0, j]} .
$$

The classical duration concept is a powerful tool to get a rule of thumb for the effect of yield curve parallel shifts. With the key-rate durations the sensitivity of non-parallel shifts can be measured when a spot rate yield curve is applied.

\section{A forward rate duration measure for the interest rate risk}

In order to account for systematic interest rate risk when a forward rate yield curve $I^{f}$ is applied, each entry $i_{j}$ of $I^{f}$ will be considered as a single variable. For measuring the interest rate risk caused by a shift $\Delta I^{f}=\left(\Delta i_{1}, \Delta i_{2}, \ldots, \Delta i_{n}\right)$, an $n$ dimensional Taylor series approximation will be used.

We get

$$
P V\left(Z, I^{f}+\Delta I^{f}\right)-P V\left(Z, I^{f}\right) \approx \frac{\mathrm{d}}{\mathrm{d} I^{f}} P V\left(Z, I^{f}\right),
$$

where $\frac{\mathrm{d}}{\mathrm{d} I^{f}} P V\left(Z, I^{f}\right)$ denotes the total differential of $P V\left(Z, I^{f}\right)$. For the sensitivity we obtain the approximation

$$
\begin{aligned}
\frac{\Delta P V\left(Z, I^{f}\right)}{P V\left(Z, I^{f}\right)} & =\frac{P V\left(Z, I^{f}+\Delta I^{f}\right)-P V\left(Z, I^{f}\right)}{P V\left(Z, I^{f}\right)} \\
& \approx-\left(\frac{-1}{P V\left(Z, I^{f}\right)} \frac{\mathrm{d}}{\mathrm{d} I^{f}} P V\left(Z, I^{f}\right)\right) .
\end{aligned}
$$

We define

$$
D\left(Z, I^{f}\right):=\frac{-1}{P V\left(Z, I^{f}\right)} \cdot \frac{\mathrm{d}}{\mathrm{d} I^{f}} P V\left(Z, I^{f}\right)
$$

as the forward rate duration. Working out the partial derivatives concerned, an analytical representation of $D\left(Z, I^{f}\right)$ is obtained:

$$
D\left(Z, I^{f}\right)=\frac{1}{P V\left(Z, I^{f}\right)} \cdot \sum_{j=1}^{n}\left(\sum_{k=1}^{j}\left(1+i_{k}\right)^{-1} \Delta i_{k}\right) \cdot Z_{j} \cdot v^{(j)} .
$$


Using $D\left(Z, I^{f}\right)$, the interest rate risk can be approximated by

$$
P V\left(Z, I^{f}+\Delta I^{f}\right)-P V\left(Z, I^{f}\right) \approx-D\left(Z, I^{f}\right) \cdot P V\left(Z, I^{f}\right) .
$$

Note, that within the last formula the different change rates $\Delta i_{t}$ do not appear explicitly as their information is contained in $D\left(Z, I^{f}\right)$.

The forward rate duration (10), obviously, unifies the duration measures mentioned in Sect. 3. For the special case that

$$
I^{f}=(i, i, \ldots, i) \quad \text { and } \quad \Delta I^{f}=(1,1, \ldots, 1)
$$

$D\left(Z, I^{f}\right)$ in $(10)$ and $D(Z, \bar{I})$ in (5) coincide, i.e.

$$
D\left(Z, I^{f}\right)=D(Z, \bar{I}) .
$$

If spot rates $i_{[0, t]}, t=1, \ldots, n$ are concerned, set $i_{t}, t=1, \ldots, n$, according to (1) and

$$
\begin{aligned}
& \Delta i_{t}:=\frac{\partial i_{t}}{\partial i_{[0, t-1]}} \Delta i_{[0, t-1]}+\frac{\partial i_{t}}{\partial i_{[0, t]}} \Delta i_{[0, t]} \\
& =-(t-1) \cdot \frac{\left(1+i_{[0, t-1]}\right)^{-t}}{\left(1+i_{[0, t]}\right)^{-t}} \Delta i_{[0, t-1]}+t \cdot \frac{\left(1+i_{[0, t-1]}\right)^{-t+1}}{\left(1+i_{[0, t]}\right)^{-t+1}} \Delta i_{[0, t]},
\end{aligned}
$$

for $t=1, \ldots, n$. Then the equation

$$
D\left(Z, I^{f}\right)=\sum_{j=1}^{n} K R D_{j} \cdot \Delta i_{[0, j]}
$$

holds. Since forward rates can be transformed into spot rates and vice versa (Remark 1), these relations are not surprising.

Using the $n$-dimensional second-order Taylor series approximation for $P V\left(Z, I^{f}+\right.$ $\Delta I^{f}$ ) we get

$$
P V\left(Z, I^{f}+\Delta I^{f}\right)-P V\left(Z, I^{f}\right) \approx \frac{\mathrm{d}}{\mathrm{d} I^{f}} P V\left(Z, I^{f}\right)+\frac{1}{2} \cdot \frac{\mathrm{d}^{2}}{\mathrm{~d} I^{f^{2}}} P V\left(Z, I^{f}\right) .
$$

We will call

$$
\begin{aligned}
C\left(Z, I^{f}\right): & =\frac{1}{P V\left(Z, I^{f}\right)} \cdot \frac{\mathrm{d}^{2}}{\mathrm{~d} I^{f^{2}}} P V\left(Z, I^{f}\right) \\
& =\frac{1}{P V\left(Z, I^{f}\right)} \cdot \sum_{j=1}^{n} Z_{j} \cdot v^{(j)} \cdot 2 \cdot \sum_{k=1}^{j} \sum_{l=k}^{j} v_{k} \Delta i_{k} \cdot v_{l} \Delta i_{l}
\end{aligned}
$$


forward rate convexity. Hence, the sensitivity can be approximated by

$$
\frac{\Delta P V\left(Z, I^{f}\right)}{P V\left(Z, I^{f}\right)} \approx-D\left(Z, I^{f}\right)+\frac{1}{2} \cdot C\left(Z, I^{f}\right)
$$

Overall, the forward rate duration $D\left(Z, I^{f}\right)$ and the forward rate convexity $C\left(Z, I^{f}\right)$ provide tools to measure the interest rate risk and the sensitivity. It incorporates the classical duration concepts.

It is important to identify the conceptual structure of the forward rate duration approach. As an approach that allows an annual consideration of non-flat interest rates, it will turn out to be an appropriate tool for biometric risk management.

\section{Biometric risk measurement}

In an actuarial environment cash flows usually consist of premiums, pensions payments or death benefits. The prescribed payments are subject to the uncertainty of life and death. Premiums and pensions payments cease upon the death of the insured person. Therefore, the dates of the prescribed payments, as well as the dates of the death benefits, are uncertain. To take these uncertainties into account annual mortality rates are applied to compute expected values of the respective payments.

In the following, we will consider an insurance contract for a person aged $x$ and policy term $n$.

Usually, for such a contract (deterministic) mortality rates are given by a life table $Q_{x}=\left(q_{x}, q_{x+1}, \ldots, q_{x+n}\right)$. Here $q_{x+t}$ is the probability of a person aged $x$ to die at the age of $x+t$. Furthermore, the probability ${ }_{t} p_{x}$ for a person of age $x$ to be alive at the beginning of age $x+t$ is calculated by

$$
{ }_{t} p_{x}=\prod_{k=0}^{t-1}\left(1-q_{x+k}\right) .
$$

Consistently with an annual consideration, a forward rate curve $I^{f}$ is still applied for the financial valuation of the prescribed payments $Z$. Then, the actuarial present value $a P V\left(Z, Q_{x}\right)^{3}$ of the expected cash flow $Z$ is defined by

$$
a P V\left(Z, Q_{x}\right)=\sum_{j=0}^{n}{ }_{j} p_{x} \cdot Z_{j} \cdot v^{(j)} .
$$

(see e.g. Promislow 2011)

\footnotetext{
${ }^{3}$ The notation $a P V\left(Z, Q_{x}\right)$ is used to emphasize the dependence on $Q_{x}$. In addition, the actuarial present value still depends on $n$ and the forward rates used.
} 


\section{Example 1}

1. Let $b=\left(b_{0}, b_{1}, \ldots, b_{n-1}, 0\right)$ be a death benefit vector, including the death benefits $b_{t}$ paid at time $t+1$ for death between time $t$ and $t+1$. Moreover, by $c=$ $\left(c_{0}, c_{1}, \ldots, c_{n}\right)$ we denote an annuity benefit vector, i.e. $c_{t}$ will be paid at time $t$ for survival of the annuitant.

Both types of benefits are combined by the expected benefit $B_{t}$ of the period $[t, t+1]$

$B_{t}=c_{t}+q_{x+t} \cdot b_{t} \cdot v_{t+1}$,

with $v_{t+1}=\left(1+i_{t+1}\right)^{-1}$, for $t=0, \ldots, n-1$. The present value of the insurance benefits will then be presented by $a P V\left(B, Q_{x}\right)$.

2. Let $\pi=\left(\pi_{0}, \pi_{1}, \ldots, \pi_{n-1}, 0\right)$ be the premium vector, where $\pi_{t}$ denotes the premium payable at time $t$. For $Z_{t}=\pi_{t}, t=0,1, \ldots, n-1$ and $Z_{n}=0, a P V\left(\pi, Q_{x}\right)$ describes the actuarial present value of the prescribed premiums. $\diamond$

For the calculation of long term contracts it is difficult to quantify $Q_{x}$ precisely in advance. Obviously, medical progress, diseases, climate change etc. essentially influence these rates over time and lead to deviations from the assumed life table $Q_{x}$. Let $\Delta Q_{x}$ qualify these deviations. Hence, there is a risk caused by a shift of the life table $Q_{x}$.

If such a shift of $Q_{x}$ is assumed, the valuation of prescribed payments will incorrect. This impact on the actuarial present value causes the biometric risk.

If the life expectancy of pensioners and policy holders increases $\left(\Delta Q_{x}<0\right)$, the risk is often referred to as longevity risk. In contrary, the term mortality risk is used when mortality rates rise $\left(\Delta Q_{x}>0\right)^{4}$. The biometric risk to which we refer covers these risks $\left(\Delta Q_{x} \neq 0\right)^{5}$.

In order to measure the biometric risk, consider the mortality rates $q_{x}$ more detailed. Usually, these entries of $Q_{x}$ are induced by a degradation of a population of size $\ell_{x}$. The mortality rates are then obtained by

$$
q_{x+t}=\frac{\ell_{x+t}-\ell_{x+t+1}}{\ell_{x+t}}, \quad t=0,1, \ldots, n,
$$

i.e. the develpoment of $\ell_{x}$ can be formulated by

$$
\ell_{x+t+1}=\ell_{x+t} \cdot\left(1-q_{x+t}\right), \quad t=0,1, \ldots, n .
$$

\footnotetext{
${ }^{4}$ These terms differs across the literature. Longevity risk also often refers to systematic deviations from a life table and is non-diversifiable, while mortality risk refers to diversifiable mortality risk that can be fully removed by signing sufficiently many contracts (Visco 2006).

${ }^{5}$ In addition to mortality rates, there are other biometric quantities that are needed in the calculation of life insurance policies. Disability probabilities, for example, are used to calculate occupational disability insurances. Changes in these quantities also cause a risk, which can be subsumed under the term biometric risk.
} 

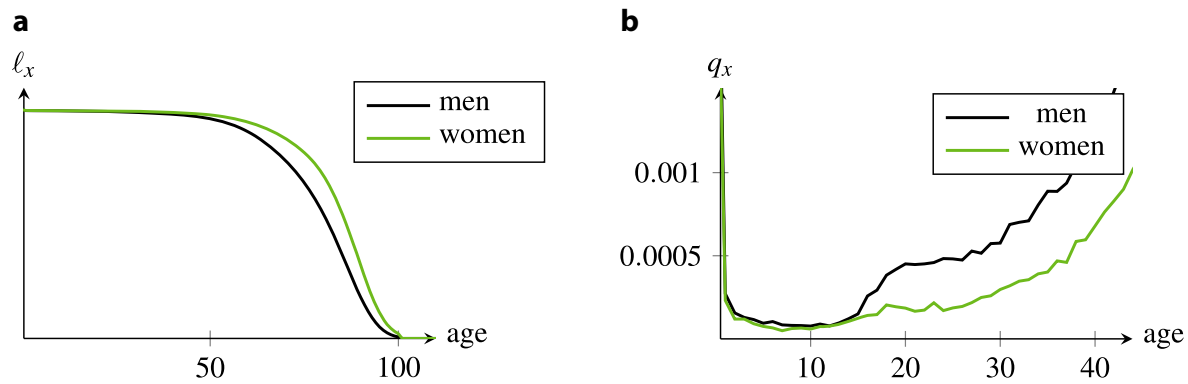

Fig. 3 Extract from a life table in accordance with the general German life table 2014/16, separately for men and women (Statistisches Bundesamt 2018). a Number $\ell_{x}$ of age $x$ survivors, separately for men and women. b Mortality rates $q_{x}$, separately for men and women

Fig. 3 shows the numbers $\ell_{x}$ of age $x$ survivors and the corresponding mortality rates $q_{x}$, all data in accordance with the general German life table ADSt2014/16, published by the German Federal Statistical Office.

It can be observed that there are significant similarities between interest rate and mortality modelling, especially when stochastic processes are applied. Using these similarities, tools and techniques developed for interest rate modelling are translated for modelling forward death intensities, e.g. by Biffis (2005); Dahl (2004); Jalen and Mamon (2009); Milevsky and Promislow (2001); Cairns et al. (2006); Luciano et al. (2012).

Beside these approachses also significant structural similarities between interest rates and mortality intensities can be observed in a deterministic setting. This structural analogy might be applied in practical actuarial environment, since deterministic interest rates and mortalities are typically used by practising actuaries.

In the view of (15), $\left(1-q_{x+t}\right)$ describes the rate of degradation of a population, just as $\left(1+i_{t}\right)$ describe the accumulation factor for a monetary value. In such a sense $-q_{x+t}$ can be interpreted as an annual "negative interest rate". As $\ell_{x+t}$ is monotonously decreasing (see Fig. 3a), $-1<-q_{x+t}<0$ holds. Within this interpretation $t p_{x}=\prod_{k=0}^{t-1}\left(1-q_{x+k}\right)$ becomes the accumulation factor corresponding to $v^{(t)^{-1}}=\prod_{j=1}^{t}\left(1+i_{j}\right)$ in a monetary environment.

The mortality rates $Q_{x}$ thus describe the successive annual degradation of a population. Due to its annual perspective, they can be interpreted as "forward rates", i.e. $Q_{x}$ becomes a "forward rate yield curve".

There are, of course, differences between interest rates and mortalities. While the assumption of a flat yield curve $\bar{I}$ may have practical benefits or relevance in a financial environment, it does not make sense for $Q_{x}$ in any case. Mortality rates vary with age $x$, they rise for high ages and are also not necessarily monotone for low ages (see Fig. 3b).

Furthermore, the consideration of a parallel shift of $Q_{x}$ is not a meaningful assumption, since changes in mortality do not affect all age groups by the same increment. 
However, taking the structural analogies into account, it is possible to transfer the forwad rate duration concept of Chapt. 4 to the management of biometric risks. From the formal point of view changes in the life table $Q_{x}$ might have similar effects on $\operatorname{aPV}\left(Z, Q_{x}\right)$ as a (non-parallel) change in a yield curve $I^{f}$ has effects on $P V\left(Z, I^{f}\right)$.

More precise, consider the actuarial present value $\operatorname{aPV}\left(Z, Q_{x}+\Delta Q_{x}\right)$ caused by a shift $\Delta Q_{x}$ of the life table $Q_{x}$. Formula (8) with respect to $Q_{x}$ instead of $I^{f}$, gives an approximation of the biometric risk:

$$
\operatorname{aPV}\left(Z, Q_{x}+\Delta Q_{x}\right)-a P V\left(Z, Q_{x}\right) \approx \frac{\mathrm{d}}{\mathrm{d} Q_{x}} a P V\left(Z, Q_{x}\right) .
$$

Transforming to the biometric sensitivity yields

$$
\frac{\Delta a P V\left(Z, Q_{x}\right)}{a P V\left(Z, Q_{x}\right)} \approx \frac{1}{a P V\left(Z, Q_{x}\right)} \cdot \frac{\mathrm{d}}{\mathrm{d} Q_{x}} \operatorname{aPV}\left(Z, Q_{x}\right) .
$$

The biometric duration $D\left(Z, Q_{x}\right)$ then will be defined as

$$
D\left(Z, Q_{x}\right):=\frac{-1}{a P V\left(Z, Q_{x}\right)} \cdot \frac{\mathrm{d}}{\mathrm{d} Q_{x}} \operatorname{aPV}\left(Z, Q_{x}\right) .
$$

For specified payments $Z$ an analytical representation for $D\left(Z, Q_{x}\right)$ is obtained by

$$
\begin{aligned}
& D\left(Z, Q_{x}\right)=\frac{-1}{a P V\left(Z, Q_{x}\right)} \cdot \frac{\mathrm{d}}{\mathrm{d} Q_{x}} a P V\left(Z, Q_{x}\right) \\
& =\frac{-1}{a P V\left(Z, Q_{x}\right)} \cdot \sum_{j=0}^{n-1} \frac{\partial a P V\left(Z, Q_{x}\right)}{\partial q_{x+j}} \Delta q_{x+j} \\
& =\frac{1}{a P V\left(Z, Q_{x}\right)} \cdot \sum_{j=1}^{n}\left(\sum_{k=0}^{j-1}\left(1-q_{x+k}\right)^{-1} \Delta q_{x+k}\right) \cdot{ }_{j} p_{x} \cdot Z_{j} \cdot v^{(j)} .
\end{aligned}
$$

The structural similarity between $D\left(Z, I^{f}\right)$ in (10) and $D\left(Z, Q_{x}\right)$ in (17) is obvious. Mortality rates, considered as negative forward rates, have the same analytical effect on the respective risk measurements.

Example 2 Consider the benefit $B_{t}$ from Example 1 and its present value

$$
\operatorname{aPV}\left(B, Q_{x}\right)=\sum_{j=0}^{n} j_{x} \cdot B_{j} \cdot v^{(j)}=\sum_{j=0}^{n}{ }_{j} p_{x} \cdot\left(c_{j}+q_{x+j} \cdot b_{j} \cdot v_{j+1}\right) \cdot v^{(j)},
$$


the biometric duration can explicitly be represented by

$$
\begin{aligned}
& D\left(B, Q_{x}\right)= \frac{-1}{a P V\left(B, Q_{x}\right)} \cdot \frac{\mathrm{d}}{\mathrm{d} Q_{x}} a P V\left(B, Q_{x}\right) \\
&= \frac{-1}{a P V\left(B, Q_{x}\right)} \cdot\left(\sum _ { k = 0 } ^ { n - 1 } \frac { \partial } { \partial q _ { x + k } } \left(\sum_{j=0}^{n}{ }_{j} p_{x} \cdot c_{j} \cdot v^{(j)}\right.\right. \\
&\left.\left.+\sum_{j=0}^{n}{ }_{j} p_{x} \cdot q_{x+j} \cdot b_{j} \cdot v^{(j+1)}\right) \Delta q_{x+k}\right) \\
&= \frac{-1}{a P V\left(B, Q_{x}\right)} \cdot\left(\sum _ { k = 0 } ^ { n - 1 } \left(\sum_{j=k+1}^{n}-\left(1-q_{x+k}\right)^{-1} \cdot{ }_{j} p_{x} \cdot c_{j} \cdot v^{(j)}\right.\right. \\
&+{ }_{k} p_{x} \cdot b_{k} \cdot v^{(k+1)} \\
&\left.\left.+\sum_{j=k+1}^{n}-\left(1-q_{x+k}\right)^{-1} \cdot{ }_{j} p_{x} \cdot q_{x+j} \cdot b_{j} \cdot v^{(j+1)}\right) \Delta q_{x+k}\right) \\
&=\frac{1}{a P V\left(B, Q_{x}\right)} \cdot\left(\sum_{j=1}^{n}\left(\sum_{k=1}^{j}\left(1-q_{x+k-1}\right)^{-1} \Delta q_{x+k-1}\right) \cdot{ }_{j} p_{x} \cdot B B_{j} \cdot v^{(j)}\right. \\
&\left.-\sum_{j=0}^{n}{ }_{j} p_{x} \cdot b_{j} \cdot v^{(j+1)} \cdot \Delta q_{x+j}\right) \cdot
\end{aligned}
$$

As the benefits $B_{t}$ also depend on $i_{t+1}$, the forward rate duration is

$$
\begin{array}{r}
D\left(B, I^{f}\right)=\frac{1}{a P V\left(B, Q_{x}\right)} \cdot\left(\sum_{j=1}^{n}\left(\sum_{k=1}^{j}\left(1+i_{k}\right)^{-1} \Delta i_{k}\right) \cdot{ }_{j} p_{x} \cdot B_{j} \cdot v^{(j)}\right. \\
\left.-\sum_{j=0}^{n}{ }_{j} p_{x} \cdot q_{x+j} \cdot b_{j} \cdot v^{(j+1)} \cdot v_{j+1} \cdot \Delta i_{j+1}\right) .
\end{array}
$$

In actuarial practice, risk measurement is of particular importance when considering the actuarial reserve.

Example 3 For $t=0, \ldots, n-1$, the actuarial reserve ${ }_{t} V_{x}$ can be expressed as

$$
{ }_{t} V_{x}=a P V_{t}(B)-a P V_{t}(\pi) .
$$


where $a P V_{t}(B)$ and $\left.a P V_{t}(\pi)\right)$ denotes the value at time $t$ of expected future benefits and premiums, respectively. It is

$$
\begin{aligned}
{ }_{t} V_{x} & =\sum_{j=0}^{n} j p_{x+t} \cdot B_{t+j} \cdot v_{t}^{(t+j)}-\sum_{j=0}^{n} j p_{x+t} \cdot \pi_{t+j} \cdot v_{t}^{(t+j)} \\
& =\sum_{j=0}^{n} j p_{x+t} \cdot \underbrace{\left(B_{t+j}-\pi_{t+j}\right)}_{=: Z_{t+j}} \cdot v_{t}^{(t+j)} \\
& =\sum_{j=0}^{n} j p_{x+t} \cdot\left(\left(c_{t+j}-\pi_{t+j}\right)+q_{x+t+j} \cdot b_{t+j} \cdot v_{t+j+1}\right) \cdot v_{t}^{(t+j)} .
\end{aligned}
$$

Therefore, the actuarial reserve is a present value of the cash flow ${ }_{t} Z=$ $\left(Z_{t}, Z_{t+1}, \ldots, Z_{n}\right)$ with term $n-t+1$. This cash flow induces a truncated forward rate curve ${ }_{t} I^{f}=\left(i_{t+1}, \ldots, i_{n}\right)$ and a truncated life table ${ }_{t} Q_{x}=\left(q_{x+t}, \ldots, q_{x+n-1}\right)$.

For measuring the biometric risk (with respect to the actuarial reserve) at time $t$, the corresponding biometric duration will be obtained by

$$
\begin{aligned}
D\left({ }_{t} Z,{ }_{t} Q_{x}\right)=\frac{1}{{ }_{t} V_{x}} \cdot( & \sum_{j=1}^{n-t}\left(\sum_{k=0}^{j-1}\left(1-q_{x+t+k}\right)^{-1} \Delta q_{x+t+k}\right) \cdot{ }_{j} p_{x+t} \cdot Z_{t+j} \cdot v_{t}^{(t+j)} \\
& \left.-\sum_{j=0}^{n}{ }_{j} p_{x+t} \cdot b_{t+j} \cdot v_{t}^{(t+j+1)} \cdot \Delta q_{x+t+j}\right) .
\end{aligned}
$$

Analogously the forward rate duration for measuring the interest rate risk is obtained by

$$
\begin{aligned}
D\left({ }_{t} Z,{ }_{t} I^{f}\right)=\frac{1}{{ }_{t} V_{x}} & \left(\sum_{j=1}^{n-t}\left(\sum_{k=1}^{j} v_{t+k} \Delta i_{t+k}\right) \cdot{ }_{j} p_{x+t} \cdot Z_{t+j} \cdot v_{t}^{(t+j)}\right. \\
& \left.+\sum_{j=0}^{n}{ }_{j} p_{x+t} \cdot b_{t+j} \cdot q_{x+t+j} \cdot v_{t}^{(t+j+1)} \cdot v_{t+j+1} \cdot \Delta i_{t+j+1}\right) .
\end{aligned}
$$

This duration can be computed explicitly for the existing calculation bases, if the expected changes are simulated and known. Therefore it should give a remarkable impact to risk management of insurance companies. $\diamond$

Similar to the forward rate convexity, a second order term can be used to improve the approximation of $\Delta a P V\left(Z, Q_{x}\right)$. We define the biometric convexity

$$
C\left(Z, Q_{x}\right):=\frac{1}{a P V\left(Z, Q_{x}\right)} \cdot \frac{\mathrm{d}^{2}}{\mathrm{~d}^{2} Q_{x}} \operatorname{aPV}\left(Z, Q_{x}\right) .
$$


With the biometric duration $D\left(Z, Q_{x}\right)$ and the biometric convexity $C\left(Z, Q_{x}\right)$ we get the approximation of the biometric risk

$$
\begin{aligned}
\operatorname{aPV}\left(Z, Q_{x}+\Delta Q_{x}\right)-\operatorname{aPV}\left(Z, Q_{x}\right) \approx & -D\left(Z, Q_{x}\right) \cdot a P V\left(Z, Q_{x}\right) \\
& +\frac{1}{2} \cdot C\left(Z, Q_{x}\right) \cdot a P V\left(Z, Q_{x}\right) .
\end{aligned}
$$

The sensitivity can be approximated by

$$
\frac{\Delta a P V\left(Z, Q_{x}\right)}{a P V\left(Z, Q_{x}\right)} \approx-D\left(Z, Q_{x}\right)+\frac{1}{2} \cdot C\left(Z, Q_{x}\right) .
$$

For a given set of mortality changes $\Delta q_{x+t}, t=0, \ldots, n-1$ the biometric convexity $C\left(Z, Q_{x}\right)$ is

$$
\begin{aligned}
C\left(Z, Q_{x}\right)= & \frac{1}{a P V\left(Z, Q_{x}\right)} \cdot \sum_{j=0}^{n}{ }_{j} p_{x} \cdot Z_{j} \cdot v^{(j)} \cdot 2 \\
& \cdot \sum_{k=0}^{j-2} \frac{1}{1-q_{x+k}} \cdot \Delta q_{x+k} \cdot \sum_{l=k+1}^{j-1} \frac{1}{1-q_{x+l}} \cdot \Delta q_{x+l} .
\end{aligned}
$$

With the concept presented additive changes of type $Q_{x}+\Delta Q_{x}$ are modelled. Frequently, however, changes of type $Q_{x}^{\prime}=Q_{x} \cdot \exp (\Delta)$ are assumed to be reasonable. Even if $\Delta$ is constant within this assumption, this will lead to a non-parallel change of the mortality rates.

Example 4 A change of type $Q_{x}^{\prime}=Q_{x} \cdot \exp (\Delta)$ is typical, when considering mortality rates depending on the year of birth $\tau$ of a person aged $x$.

For a basis year $\tau_{0}$ a life table $Q_{x}^{\left(\tau_{0}\right)}=\left(q_{x}^{\left(\tau_{0}\right)}, q_{x+1}^{\left(\tau_{0}\right)}, \ldots, q_{x+n}^{\left(\tau_{0}\right)}\right)$ is given. The life table for a person aged $x$ and born in the year $\tau$ is denoted by $Q_{x}^{(\tau)}=$ $\left(q_{x}^{(\tau)}, q_{x+1}^{(\tau)}, \ldots, q_{x+n}^{(\tau)}\right)$. Here $q_{x+t}^{(\tau)}$ reflects the corresponding mortality probabilities. The mortality rates $q_{x+t}^{(\tau)}$ are typically modelled by

$$
q_{x+t}^{(\tau)}=q_{x+t}^{\left(\tau_{0}\right)} \cdot \exp \left(F(x) \cdot\left(\tau_{0}-(\tau+x+t)\right)\right) .
$$

In DAV Arbeitsgruppe (2005) $\tau_{0}=1999$ is used and $F(x)$ is an age-dependent trend function.

Setting $Q_{x}=Q_{x}^{\left(\tau_{0}\right)}$ and $Q_{x}^{\prime}=Q_{x}^{(\tau)}$ the model is of type $Q_{x}^{\prime}=Q_{x} \cdot \exp (\Delta)$. Obviously, $\Delta$ is not constant in this case.

Using $\Delta Q_{x}=Q_{x}^{(\tau)}-Q_{x}^{\left(\tau_{0}\right)}$, the biometric risk can be measured by the biometric duration approach when mortality rates $Q_{x}^{(\tau)}$ depending on the year of birth are used instead of the basis life table $Q_{x}^{\left(\tau_{0}\right)}$. $\diamond$ 
Fig. 4 Extract from a life table in accordance with the life table DAV2004R, separately for men, women and unisex

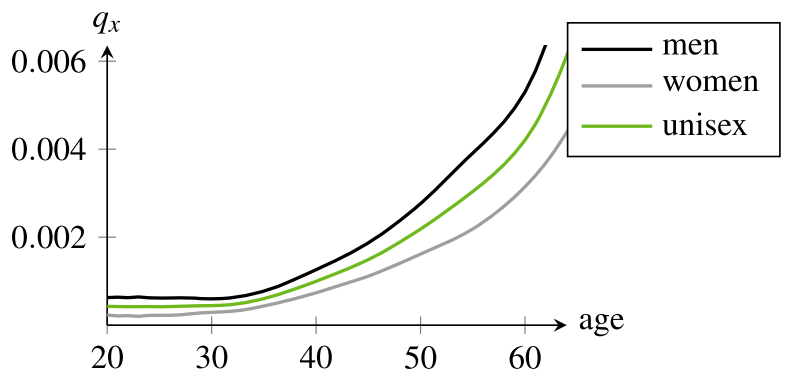

Since 2012 it has been forbidden in European countries to include the gender of the insured person in calculation. This provides an example for the use of the biometric duration.

Example 5 The adjustment of the different gender life tables $Q_{x}$ and $Q_{y}$ to unisex tables $Q_{x y}$ for new life insurance contracts, can also be used as an example for a change of mortality rates. We use the weighted average of the gender specific life tables $Q_{x}$ and $Q_{y}$, the weighting is based on the number of living men and women (Ortmann 2016). We get for the unisex death probabilities ${ }^{6}$ :

$$
q_{x y+t}:=\frac{\ell_{x+t}}{\ell_{x+t}+\ell_{y+t}} \cdot q_{x+t}+\frac{\ell_{y+t}}{\ell_{x+t}+\ell_{y+t}} \cdot q_{y+t}, \quad t=0,1, \ldots, n .
$$

Fig. 4 shows the mortality rates in accordance to the life tables DAV2004R for men and women, respectively, published by the German actuarial association (DAV Arbeitsgruppe 2005).

Using the unisex table $Q_{x y}$, there will, of course, be a deviation $\Delta Q_{x y}$ from the gender specific life table $Q_{x}$, i.e. $Q_{x}-Q_{x y}=\Delta Q_{x y}$. Therefore inaccuracies in the calculation will occur. The changes $\Delta q_{x y+t}$ are

$$
\Delta q_{x y+t}=q_{x+t}-q_{x y+t}=\left(q_{x+t}-q_{y+t}\right) \cdot \frac{\ell_{y+t}}{\ell_{x+t}+\ell_{y+t}}, \quad t=0, \ldots, n .
$$

We use the corresponding data for a life annuity on the life of a male person aged $x=32$. Constant annual premiums are payable for 35 years until the pensions payments will start. Then the annuitant will get an annuity of $1000 €$ per year for the next 30 years or as long as he is alive.

We assume a flat yield curve with interest $i=0.9 \%$. For this insurance, the annual premium is $\pi_{t}=€ 412.26, t=0, \ldots, 34$. For the actuarial reserve ${ }_{35} V_{32}\left(Q_{x y}\right)$ at the beginning of the annuity payments with ${ }_{35} Z=(1000, \ldots, 1000,0)$, we get ${ }_{35} V_{32}\left(Q_{x y}\right)=€ 18193.06$. Here, the biometric duration equals $D\left({ }_{35} Z, Q_{x y}\right)=$ 0.0756 . For the biometric convexity we get $C\left({ }_{35} Z, Q_{x y}\right)=0.00021$.

\footnotetext{
${ }^{6} Q_{x}, Q_{y}$ and $Q_{x y}$ denote the life tables for men, women and the unisex life table, respectively, and contain the mortality rates for the same ages.
} 
The biometric risk can be approximated by

$$
\begin{aligned}
{ }_{35} V_{32}\left(Q_{x}\right)-{ }_{35} V_{32}\left(Q_{x y}\right) \approx & -D\left({ }_{35} Z, Q_{x y}\right) \cdot{ }_{35} V_{32}\left(Q_{x y}\right) \\
& +\frac{1}{2} C\left({ }_{35} Z, Q_{x y}\right) \cdot{ }_{35} V_{32}\left(Q_{x y}\right) \\
= & -€ 1372.96
\end{aligned}
$$

and the sensitivity by

$$
\begin{aligned}
\frac{\Delta_{35} V_{32}\left(Q_{x y}\right)}{{ }_{35} V_{32}\left(Q_{x y}\right)} & \approx-D\left({ }_{35} Z, Q_{x y}\right)+\frac{1}{2} C\left({ }_{35} Z, Q_{x y}\right) \\
& =-0.07547 .
\end{aligned}
$$

Hence, the necessary actuarial reserve is approximately $7.55 \%$ smaller than the calculated one. Since men share the longevity risk of women in unisex pension insurance, this result has to be expected. $\diamond$

\section{Conclusion}

For the financial sector classical duration concepts provide various instruments for risk assessment. These approaches typically use Taylor-series approximation of the present value of cash flows.

For long term insurance contracts not only the interest rate risk is of essential importance. Also the risk caused by disturbances in biometric data must taken into consideration. Such disturbances may come from medical progress and longevity, unknown lethal diseases, the appearance of epidemics and so on. In order to take account of these uncertainties, the paper presented an approach that is suitable for daily actuarial practice. Its idea is based on typical techniques used in financial risk management. So, for its application no additional or extraordinary mathematics is necessary.

Regarding the data of a life table as a type of negative interest rates the biometric duration and the biometric convexity was defined. They were applied to actuarial present values. Also the actuarial reserve, as a special present value, was considered in order to prevent a comprehensive tool for practising actuaries.

The uncertainties in biometric data have an essential impact on the calculation of life insurance contracts. With the biometric duration and convexity the resulting risks can be adequately considered and controlled.

Acknowledgements The authors would like to thank the anonymous reviewers for their valuable comments and suggestions.

Open Access This article is licensed under a Creative Commons Attribution 4.0 International License, which permits use, sharing, adaptation, distribution and reproduction in any medium or format, as long as you give appropriate credit to the original author(s) and the source, provide a link to the Creative Commons licence, and indicate if changes were made. The images or other third party material in this article are included in the article's Creative Commons licence, unless indicated otherwise in a credit line 
to the material. If material is not included in the article's Creative Commons licence and your intended use is not permitted by statutory regulation or exceeds the permitted use, you will need to obtain permission directly from the copyright holder. To view a copy of this licence, visit http://creativecommons.org/ licenses/by/4.0/.

Funding Open Access funding provided by Projekt DEAL.

\section{References}

Biffis, E.: Affine processes for dynamic mortality and actuarial valuations. Insur. Math. Econ. 37(3), 443-468 (2005)

Bühler, A., Hies, M.: Zinsrisiken und Key-Rate-Duration. Die Bank 2, 112-118 (1995)

Cairns, A.J.G., Blake, D., Dowd, K.: Pricing Death: frameworks for the valuation of securitization of mortality risk. ASTIN Bull. 36(1), 79-120 (2006)

Coughlan, G.: Longevity Risk Transfer: Indices and Capital Market Solutions. The Handbook of InsuranceLinked Securities, Wiley, New York, pp 261-281 (2012)

Coughlan, G., Epstein, D., Ong, A., Sinha, A., Hevia-Portocarrero, J., Gingrich, E., Khalaf-Allah, M., Joseph, P.: LifeMetrics: A toolkit for measuring and managing longevity and mortality risks. JPMorgan Securities Ltd, Technical Report, www.lifemetrics.com (2007)

Dahl, M.: Stochastic mortality in life insurance: market reserves and mortality-linked insurance contracts. Insur. Math. Econ. 35(1), 113-136 (2004)

DAV Unterarbeitsgruppe Rentnersterblichkeit: Herleitung der DAV-Sterbetafel 2004 R für Rentenversicherungen. Blätter der DGVFM 27(2), 199-314 (2005)

Fabozzi, F.J., Mann, S.V., Choudhry, M.: Measuring and Controlling Interest Rate and Credit Risk. John Wiley \& Sons, New Jersey (2003)

Fisher, L., Weil, R.L.: Coping with the risk of interest-rate fluctuations. J. Bus. 44(4), 408-431 (1971)

Fong, C., Vasicek, O.: A risk minimizing strategy for portfolioimmunization. J. Finance 39, 1541-1546 (1984)

Hicks, J.R.: Value and Capital: An Inquiry into some Fundamental Principles of Economic. Clarendon, Oxford (1939)

Ho, T.: Key rate durations: measures of interest rate risks. J. Fixed Income 2, 29-44 (1992)

Jalen, L., Mamon, R.: Valuation of contingent claims with mortality and interest rate risks. Math Comput Model 49(9-10), 1893-1904 (2009)

Li, J., Luo, A.: Key Q-duration: a framework for hedging longevity risk. ASTIN Bull. 42(2), 413-452 (2012)

Luciano, E., Regis, L., Vigna, E.: Delta-gamma hedging of mortality and interest rate risk. Insur. Math. Econ. 50(3), 402-412 (2012)

Macaulay, F.R.: Some Theoretical Problems Suggested by the Movements of Interest Rates, Bond Yields and Stock Prices in the United States since 1856. National Bureau of Economic Research, New York, pp 573-591 (1938)

Milevsky, M.A., Promislow, S.D.: Mortality derivatives and the option to annuitise. Insur. Math. Econ. 29(3), 299-318 (2001)

Ortmann, K.M.: Praktische Lebensversicherungsmathematik. Springer Spektrum, Wiesbaden (2016)

Plat, R.: One-year value-at-risk for longevity and mortality. Insur. Math. Econ. 49(3), 462-470 (2011)

Promislow, S.D.: Fundamentals of Actuarial Mathematics. John Wiley \& Sons, United Kingdom (2011)

Schich, S.: Schätzung der deutschen Zinsstrukturkurve. Bundesbank Series 1 Discussion Paper No.04. (1997)

Statistisches Bundesamt: Sterbetafel 2014/2016 - Ergebnisse aus der laufenden Berechnung von Periodensterbetafeln für Deutschland und die Bundesländer. www.destatis.de, Accessed 30 Aug 2018

Trautmann, S.: Investitionen. Springer, Berlin Heidelberg (2007)

Tuckman, B., Serrat, A.: Fixed Income Securities: Tools For Today's Markets. John Wiley \& Sons, New Jersey (2011)

Visco, I.: Longevity risk and financial markets. In: Balling, M., Gnan, E., Lierman, F. (eds.) Money, finance and demography: the consequences of ageing. SUERF colloquium volume, SUERF, Vienna (2006)

Wang, J.L., Huang, H.C., Yang, S.S., Tsau, J.T.: An optimal product mix for hedging longevity risk in life insurance companies: the immunization theory approach. J. Risk Insur. 77(2), 473-497 (2010) 